\title{
Aplicação do método mexicano de ordenamento florestal (MMOF) para estimar ciclo e intensidade de corte em floresta na Amazônia oriental
}

\author{
Application of the Mexican method of forest planning (MMFP) to \\ estimate the cutting cycle and intensity in the eastern Amazon rainforest
}

Leonardo Pequeno Reis', Agostinho Lopes de Souza ${ }^{2}$, Pamella Carolline Marques dos Reis ${ }^{3}$, Lucas José Mazzei de Freitas ${ }^{4}$, Liniker Fernandes da Silva ${ }^{5}$ e Lyvia Julienne Sousa Rego ${ }^{3}$

\section{Resumo}

A intensidade de corte (IC) e o ciclo de corte (CC) em florestas tropicais na Amazônia são parâmetros fundamentais para a regulação sustentável da produção de madeiras. O trabalho teve como objetivo avaliar a utilização do Método Mexicano de Ordenamento Florestal (MMOF) na Amazônia Oriental. O estudo foi realizado no município de Moju, estado do Pará (020 08'14" S e $48^{\circ} 47^{\prime} 34^{\prime \prime}$ W, ponto central), em uma área submetida a exploração de impacto reduzido (EIR), com IC de $21 \mathrm{~m}^{3} \mathrm{ha}^{-1}$. Foi avaliado o MMOF por meio do CC, IC e o volume estimado comparado com a média dos dados observados em 22 parcelas permanentes de 0,5 ha, para os anos de 1998 ( 1 ano após a colheita) e 2010 (13 anos após a colheita). Os CCs calculados para o grupo de espécies comerciais e separadamente para a espécie comercial ManiIkara huberi (Ducke) A. Chev., respectivamente, foram 22 e 19 anos para a maior taxa de crescimento $\left(i_{3}\right)$, ficando dentro do recomendado para florestas tropicais. A IC calculada $\left(31,888 \mathrm{~m}^{3} \mathrm{ha}^{-1}\right)$ para um CC de 35 anos também ficou próximo ao recomendado para florestas tropicais na Amazônia (20 a $\left.40 \mathrm{~m}^{3} \mathrm{ha}^{-1}\right)$. Os volumes estimados para os anos 1998 e 2010 não se diferenciaram do observado nas parcelas $(p>0,05)$. O MMOF pode ser usado para indicar a intensidade de corte (IC) e o ciclo de corte (CC) em florestais na Amazônia Oriental.

Palavras-chave: Floresta inequiânea, Regulação florestal, Colheita seletiva.

\section{Abstract}

The cutting intensity $(\mathrm{Cl})$ and the cutting cycle $(\mathrm{CC})$ in tropical forests in the Amazon are critical parameters for the regulation of sustainable wood production. This study aimed to evaluate the use of the Mexican Method of Forest Planning (MMFP) in the Eastern Amazon. The study was conducted in the municipality of Moju, Pará State $\left(02^{\circ} 08^{\prime} 14^{\prime \prime} S\right.$ and $\left.48^{\circ} 47^{\prime} 34^{\prime \prime} \mathrm{W}\right)$, in an area subjected to reduced impact logging (RIL), with IC $21 \mathrm{~m}^{3}$ ha $^{-1}$ Manilkara huberi (Ducke) A. Chev. were 22 and 19 years, respectively, for the highest growth rate (i3), which falls within the recommended for tropical forests. The calculated IC $\left(31,888 \mathrm{~m}^{3} \mathrm{ha}^{-1}\right)$ for a CC of 35 years was also close to the recommended for tropical forests in the Amazon (20 to $\left.40 \mathrm{~m}^{3} \mathrm{ha}^{-1}\right)$. The estimates for the years 1998 and 2010 did not differ from the observed in the plots $(p>0.05)$. The MMFP can be used to indicate the cutting intensity $(\mathrm{Cl})$ and the cutting cycle $(\mathrm{CC})$ in forest in the Eastern Amazon.

Keywords: Uneven-aged Forest, Forest Regulation, Selective Harvest.

\section{INTRODUÇÃO}

O Manejo Florestal Sustentável (MFS) apresenta-se como a atividade mais viável econômica, ecológica e social na utilização dos recursos florestais na Amazônia. Para garantir esta sustentabilidade, métodos de controle de corte, baseados na dinâmica florestal devem ser construídos para indicar a intensidade de corte (IC) e ciclos de corte (CC) mais adequados, dependendo do crescimento das espécies florestais (BRAZ et al., 2012; REIS et al., 2015).

\footnotetext{
${ }^{1}$ Doutor em Ciência Florestal. UFV - Universidade Federal de Viçosa. Campus Universitário. Av. Peter Henry Rolfs, s/n 36.570-900 - Viçosa, MG, Brasil. E-mail: leonardopequenoreis@gmail.com

2Professor Titular do Departamente de Engenharia Florestal. Universidade Federal de Viçosa. Campus Universitário. Av. Peter Henry Rolfs, s/n - 36.570-900 - Viçosa, MG, Brasil. E-mail: alsouza@ufv.br

32Doutoranda em Ciência Florestal. UFV - Universidade Federal de Viçosa. Campus Universitário. Av. Peter Henry Rolfs, s/n - 36.570-900 - Viçosa, MG, Brasil. E-mail: pamellaca@gmail.com; lyviajulienne@hotmail.com

${ }^{4}$ Pesquisador Doutor. Embrapa - Empresa Brasileira de Pesquisa Agropecuária. Trav. Dr. Éneas Pinheiro s/n - Caixa Postal 48 - 66.095-000 - Belém, PA, Brasil. E-mail: lucas.mazze@embrapa.br

${ }^{5}$ Professor Adjunto do Departamento de Engenharia Florestal. UFRB - Universidade Federal do Recôncavo da Bahia. Rua Rui Barbosa, 710 - 44380000 - Cruz das Almas, BA - Brasil. E-mail: linikerfs@gmail.com
}

Sci. For., Piracicaba, v. 45, n. 113, p. 187-195, mar. 2017 DOI: dx.doi.org/10.18671/scifor.v45n113.19 
O tempo que a floresta leva para recompor a intensidade de corte é denominado ciclo de corte. Assim, o ciclo de corte atua como instrumento regulador da quantidade de madeira removida, controlando o nível de estoque de crescimento (SOUZA et al., 2004).

A legislação que regulamenta o MFS na Amazônia (BRASIL, 2015), quando não há estudos para a área, estabelece que a produtividade anual da floresta deva ser de $0,86 \mathrm{~m}^{3} \mathrm{ha}^{-1}$.ano- ${ }^{-1}$, com isso, se a intensidade de corte for $30 \mathrm{~m}^{3} \mathrm{ha}^{-1}$ o ciclo de corte será de 35 anos. Para os cálculos de IC e CC são considerados os grupos de espécies comerciais, não havendo indicação do método de controle de corte na legislação. Braz et al. (2012) enfatizaram que o conceito manejo de florestas não estará completo, em qualquer plano de manejo, se não contiver uma previsão razoável de quanto a floresta pode recuperar dentro de um intervalo de tempo. Por isso, determinar um método de controle de corte é importante, como um guia inicial de corte nos planos de manejos florestais sustentáveis (PMFS).

No controle de corte por volume, a colheita é programada pelo volume e distribuição do estoque de crescimento e incremento. Sendo útil como um guia geral na determinação de corte, ele permite a aproximação rápida do corte admissível, com dados florestais limitados de crescimento, valorosos para um planejamento geral (SOUZA; SOARES, 2013).

Entre os diversos métodos de controle de corte por volume destaca-se o Método Mexicano de Ordenamento Florestal (MMOF), que foi proposto oficialmente em 1944 pelo Serviço Florestal Mexicano (ROJO, 2000). No MMOF o volume do estoque remanescente fundamenta-se no pressuposto de que os crescimentos anuais do estoque remanescente de uma floresta se acumulam seguindo a lei de juros compostos (SOUZA; SOARES, 2013).

O trabalho teve como objetivo avaliar a utilização do Método Mexicano de Ordenamento Florestal na Amazônia Oriental, para subsidiar a iniciativa privada e políticas públicas em relação aos planos de manejos florestais sustentáveis.

\section{MATERIAL E MÉTODOS}

O estudo foi conduzido na estação Experimental da Embrapa Amazônia Oriental, localizada no município de Moju, estado do Pará. A área total da estação é de 1.050 ha, localizada entre as coordenadas geográficas $02^{\circ} 08^{\prime} 14^{\prime \prime}$ e $02^{\circ} 12^{\prime} 26^{\prime \prime}$ de latitude sul e $48^{\circ} 47^{\prime} 34^{\prime \prime}$ e $48^{\circ} 48^{\prime} 14^{\prime \prime}$ de longitude a oeste de Greenwich (Figura 1), próximo ao km 30 da rodovia PA-150 (REIS et al., 2014 ${ }^{\mathrm{a}}$; REIS et al., 2014b).

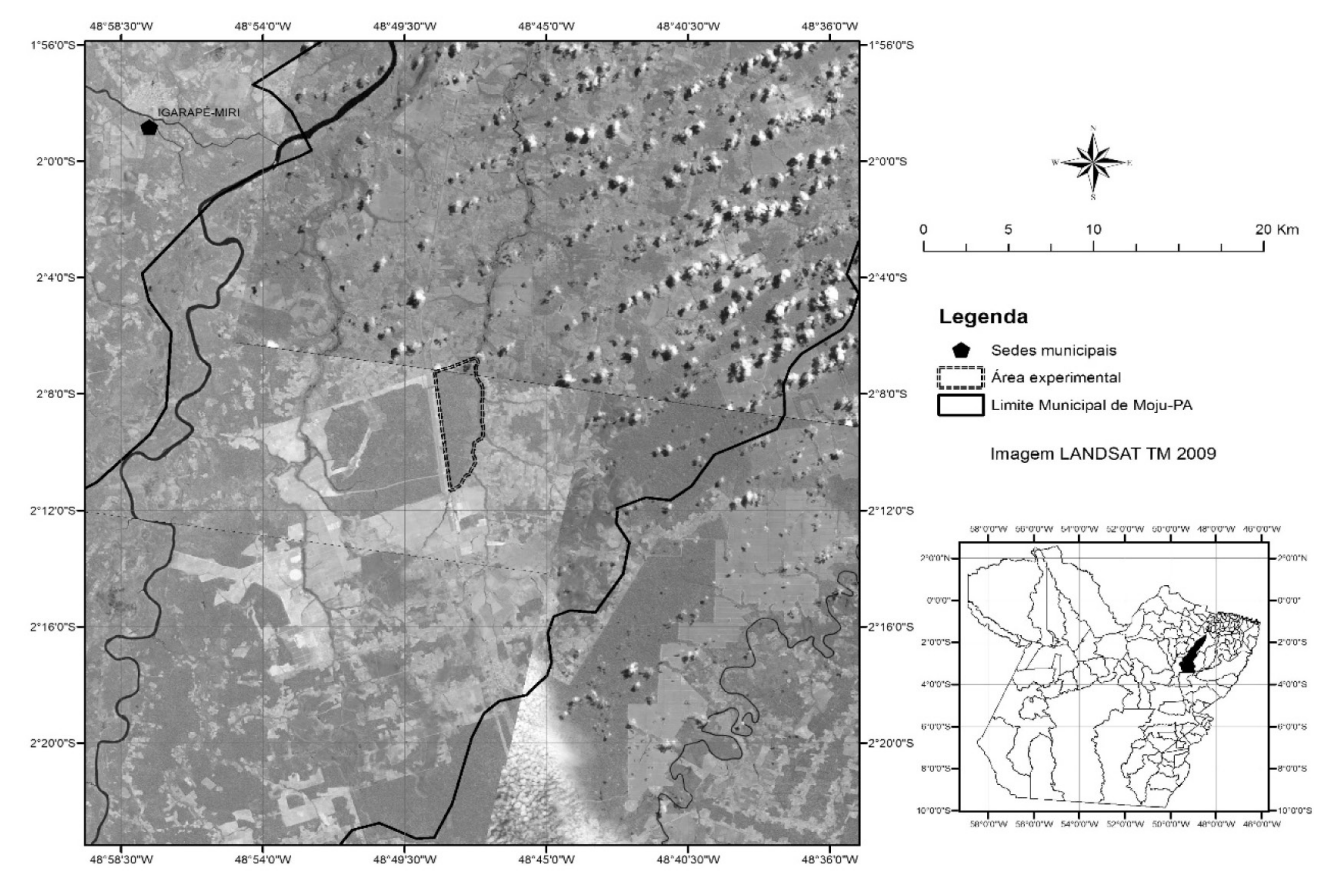

Figura 1. Localização do campo experimental da Embrapa Amazônia Oriental no município de Moju, Estado do Pará. Fonte: Reis et al. (2014a).

Figure 1. Location of the experimental field of Embrapa Amazônia Oriental in the city of Moju, Pará State. Sources: Reis et al. (2015). 
O clima da região é do tipo Ami (clima quente e úmido), segundo a classificação de Köppen. A precipitação pluviométrica anual varia de 2.000 a $3.000 \mathrm{~mm}$, distribuída irregularmente, tendo pequenos períodos secos, sendo o período mais chuvoso nos meses de fevereiro a abril, e o mais seco de agosto a outubro. A umidade relativa do ar está em torno de $85 \%$. As temperaturas médias mensais oscilam de 21 a $33{ }^{\circ} \mathrm{C}$ e a média anual é de $26^{\circ} \mathrm{C}$ (SILVA et al., 2001).

O relevo da área é plano, com pequenas ondulações. Predomina na área experimental o Latossolo Amarelo distrófico com diferentes texturas ocorrendo também solos Argissolos Vermelhos-Amarelos, Gleissolos e Plintossolo (SILVA et al., 2001), na área de efetiva colheita predomina o Latossolo Amarelo distrófico. A tipologia da área experimental é Floresta Ombrófila Densa de terra firme. Possui árvores com o porte variando entre 25 a $35 \mathrm{~m}$ de altura com presença de algumas palmeiras no sub-bosque (LOPES et al., 2001).

Em 1997, foi realizada a exploração florestal. Antes da colheita foi realizado o corte de todos os cipós com diâmetro $\geq 2 \mathrm{~cm}$ nos 200 ha. Foram colhidas, em média, 3,3 árvores ha ${ }^{-1}$ com diâmetro mínimo de corte (DMC) de $65 \mathrm{~cm}$, de 25 espécies comerciais, correspondendo a um volume de $23 \mathrm{~m}^{3}$ ha $^{-1}$, que representou 69\% do volume planejado de 33,5 $\mathrm{m}^{3} \mathrm{ha}^{-1}$ (COSTA et al., 1998; REIS et al., 2014).

Destacaram-se em volume no planejamento da colheita, Manilkara huberi (Ducke) A. Chev. com $5,8 \mathrm{~m}^{3} \mathrm{ha}^{-1}$, Vouacapoua americana Aubl.com 4,5 $\mathrm{m}^{3} \mathrm{ha}^{-1}$, Pseudopiptadenia suaveolens (Miq.) J.W. Grimes com 3,6 $\mathrm{m}^{3}$ ha-1 e Goupia glabra Aubl. com 3,1 $\mathrm{m}^{3} \mathrm{ha}^{-1}$ (Tabela 1), que juntas representaram $51 \%$ do volume planejado (SILVA et al., 2001).

Tabela 1. Espécies planejadas para colheita em 1997 de 200 ha de uma Floresta Ombrófila Densa, no município de Moju, estado do Pará. N: número de árvores planejadas para colheita; $\mathrm{m}^{3} \mathrm{ha}^{-1}$ : volume planejado para colheita por espécie.

Table 1. Planned species for harvest in 1997 of 200 ha of a dense rain forest in the municipality of Moju, Para State $\mathrm{N}$ : Number of trees to harvest; $\mathrm{m}^{3} \mathrm{ha}^{-1}$ : volume planned for harvest by species.

\begin{tabular}{llcc}
\hline Nome vulgar & Nome científico & $\mathbf{N}$ & $\mathbf{m}^{\mathbf{3}} \mathbf{h a}^{-1}$ \\
caju-açu & Anacardium giganteum W. Hancock ex Engl. & 30 & 1,10 \\
mururé & Brosimum obovata Ducke & 33 & 1,08 \\
piquiarana & Caryocar glabrum Pers. & 22 & 1,10 \\
piquiá & Caryocar villosum (Aubl.) Pers. & 10 & 0,42 \\
tauarí & Couratari oblongifolia Ducke \& R. Knuth & 42 & 1,83 \\
fava-mapuxiqui & Dimorphandra gardneriana Tul. & 19 & 0,59 \\
angelim-pedra & Dinizia excelsa Ducke & 16 & 1,12 \\
cupiúba & Goupia glabra Aubl. & 73 & 3,13 \\
angelim-vermelho & Hymenaea sp & 6 & 0,38 \\
jutaí-açú, jatobá & Hymenaea courbaril L. & 15 & 0,69 \\
angelim-da-mata & Hymenolobium excelsum Ducke & 6 & 0,23 \\
maparajuba & Manilkara bidentata (A. DC.) A. Chev. & 10 & 0,34 \\
maçaranduba & Manilkara paraensis (Huber) Standl. & 155 & 5,81 \\
louro-vermelho & Manilkara huberi (Ducke) A. Chev. & 21 & 0,86 \\
fava-barriguga & Ocotea rubra Mez & 47 & 1,82 \\
fava-bolota & Parkia gigantocarpa Ducke & 15 & 0,73 \\
angelim-rajado & Parkia pendula (Willd.) Benth. ex Walp. & 7 & 0,34 \\
saboeiro & Zigia racemosa (Ducke) Barneby \& J.W. Grimes & 14 & 0,42 \\
faveira fl. Fina & Abarema jupumba (Willd.) Britton \& Killip & 106 & 3,62 \\
mandioqueira-aspera & Pseudopiptadenia suaveolens (Miq.) J.W. Grimes & 16 & 0,77 \\
axixá & Qualea albiflora Warm. & 27 & 0,77 \\
tanimbuca & Sterculia pilosa Ducke & 34 & 1,29 \\
breu-sucuruba & Terminalia amazonia (J.F.Gmel.) Exell & 12 & 0,57 \\
acapu & Trattinickia rhoifolia Willd. & 139 & 4,54 \\
\hline Total & Vouacapoua americana Aubl. & 875 & 33,47 \\
\hline Font Edita & & 6 \\
\hline
\end{tabular}

Fonte: Edital de licitação $N^{\circ}$ 00/96 - Embrapa Amazônia Oriental e Autorização para exploração de Plano de Manejo Florestal Sustentável (PMFS) $-N^{\circ} 45 / 96$ - IBAMA.

Em 1995, foram estabelecidas, aleatoriamente, nos 200 ha escolhidos dentro da área experimental para ser realizado a colheita florestal, 22 parcelas permanentes com dimensões de $50 \times 100$ m (0,5 ha) divididas em 50 subparcelas de 10 x 10 m, totalizando uma amostra de $11 \mathrm{ha}$. Nessas 
parcelas foram medidas todas as árvores com DAP $\geq 10 \mathrm{~cm}$ em 1995 (antes da colheita) e remedidas após a colheita em 1998 e 2010 (SILVA et al., 2001).

A equação usada para obter, no Método Mexicano de Ordenamento Florestal (MMOF), o volume no tempo t em anos (ROJO, 2000) foi:

$$
\operatorname{Vn}=\operatorname{Vr}(1+i)^{c c}
$$

Sendo que, $V n=$ volume ao final do ciclo de corte, $\mathrm{em} \mathrm{m}^{3} \mathrm{ha}^{-1} ; \mathrm{Vr}=$ volume remanescente ou após o corte, em $\mathrm{m}^{3} \mathrm{ha}^{-1} ; c c=$ ciclo de corte, em anos; $i=$ taxa de crescimento.

Para a obtenção da intensidade de corte (IC) e ciclo de corte, as equações foram as seguintes:

$$
\begin{gathered}
I C=\left[1-\frac{1}{(1+i)^{c c}}\right] \cdot 100 \\
c c=\frac{\operatorname{Ln}(V n)-\operatorname{Ln}(V r)}{\operatorname{Ln}(1+i)}
\end{gathered}
$$

Sendo que, IC = Intensidade de cote, $\mathrm{em} \mathrm{m}^{3} \mathrm{ha}^{-1}$.

Para obtenção das taxas de crescimento foram utilizadas as seguintes equações da literatura (SOUZA; SOARES, 2013):

$$
i_{1}=\frac{C r}{V n} ; i_{2}=\frac{C r}{\frac{V r}{2}} ; i_{3}=\frac{C r}{V r}
$$

Sendo que, $i=$ taxa de crescimento; $\mathrm{Cr}=$ crescimento, $\mathrm{m}^{3} \mathrm{ha}^{-1} \mathrm{ano}^{-1}$.

$\mathrm{Na}$ avaliação do MMOF foram realizadas comparações do volume projetado pelo método de controle de corte com o observado pelas parcelas permanentes através do teste estatístico Wilcoxon-Mann-Whitney (independente) a 5\% de probabilidade, para os anos de 1998 e 2010.

$\mathrm{Na}$ análise foi considerado que a colheita realizada foi igual para todas as parcelas, sendo para a comunidade total e espécies comerciais de $23,00 \mathrm{~m}^{3} \mathrm{ha}^{-1} \mathrm{e}$ para a espécie $M$. huberi o volume de colheita foi de 3,198 $\mathrm{m}^{3} \mathrm{ha}^{-1}$, esse último identificado pelas parcelas permanentes. Na aplicação do MMOF foram consideradas as árvores com $\mathrm{DAP} \geq 20 \mathrm{~cm}$, medição tomada a $1,30 \mathrm{~m}$ do solo.

Os volumes de madeira foram calculados pela equação de simples entrada, específica para a área de estudo, proposta por Baima et al. (2001) para árvores em pé com $\mathrm{DAP} \geq 20 \mathrm{~cm}\left(\mathrm{R}^{2}=0,9391\right.$; índice de Furnival= 0,9093; e CV= 21,8\%):

$$
\ln V=-7,49337+2,086952 \ln d
$$

Sendo que, $\mathrm{V}$ = volume comercial com casca; $\mathrm{d}$ = DAP de cada árvore; e ln = logaritmo natural.

\section{RESULTADOS E DISCUSSÃO}

Em 1995 o volume total observado foi de 205,503 $\mathrm{m}^{3} \mathrm{ha}^{-1}$, deste, 27,8\% foram considerados com o valor comercial madeireiro, contemplando 25 espécies (Tabela 2). Dentre essas espécies $M$. huberi representou do volume 4,2\% e 15,1\%, do total da comunidade e das espécies comerciais, respectivamente.

Tabela 2. Ciclos de corte (CC em anos) calculados pelo Método Mexicano de Ordenamento Florestal (MMOF) em uma floresta de terra firme na Amazônia Oriental. Vn: Volume inicial $\left(\mathrm{m}^{3} \mathrm{ha}^{-1}\right)$; Vr: volume remanescente $\left(\mathrm{m}^{3} \mathrm{ha}^{-1}\right)$; IC: intensidade de corte $\left(\mathrm{m}^{3} \mathrm{ha}^{-1}\right)$; $\mathrm{Cr}$ : Crescimento observado $\left(\mathrm{m}^{3} \mathrm{ha}^{-1} \mathrm{ano}^{-1}\right)$; i: taxa de crescimento.

Table 2. Cutting cycles ( $C C$ in years) calculated by the Mexican Method of Forest Planning (MMFP) in a terra firme forest in Eastern Amazon. $\mathrm{Vn}$ : Initial volume $\left(\mathrm{m}^{3} \mathrm{ha}^{-1}\right)$; $\mathrm{Vr}$ : remanescent volume $\left(\mathrm{m}^{3} \mathrm{ha}^{-1}\right)$; IC: cutting intensity $\left(\mathrm{m}^{3} \mathrm{ha}^{-1}\right) ; \mathrm{Cr}$ : Observed growth $\left(\mathrm{m}^{3} \mathrm{ha}^{-1}\right.$ year $\left.{ }^{-1}\right)$; i: growth rate.

\begin{tabular}{lccccccc}
\hline Espécie & $\mathbf{V n}$ & $\mathbf{V r}$ & $\mathbf{I C}$ & $\mathbf{C r}$ & $\mathbf{C C} \mathbf{i}_{1}$ & $\mathbf{C} \mathbf{C i}_{\mathbf{2}}$ & $\mathbf{C C i}_{3}$ \\
Manilkara huberi (Ducke) A. Chev. & 8,679 & 5,481 & 3,198 & 0,133 & 30 & 25 & 19 \\
Espécies comerciais & 57,304 & 34,304 & 23,000 & 0,806 & 37 & 29 & 22 \\
Total comunidade & 205,502 & 182,502 & 23,000 & 3,212 & 8 & 7 & 7 \\
\hline
\end{tabular}

A intensidade de corte (IC) praticada na colheita representou $11,2 \%$ para o volume total da comunidade, $40,1 \%$ para o grupo de espécies comerciais e $36,1 \%$ para a $M$. huberi (Tabela 2). O total 
da comunidade obteve um crescimento mais elevado $\left(3,122 \mathrm{~m}^{3} \mathrm{ha}^{-1}\right.$ ano $\left.{ }^{-1}\right)$ em relação ao grupo de espécies comerciais $\left(0,806 \mathrm{~m}^{3} \mathrm{ha}^{-1}\right.$ ano $\left.^{-1}\right)$ e da M. huberi $\left(0,133 \mathrm{~m}^{3} \mathrm{ha}^{-1} \mathrm{ano}^{-1}\right)$.

Os ciclos de corte estimados pelo MMOF, considerando o total da comunidade, foram menores $\left(\mathrm{CC} i_{1}=8 ; \mathrm{CC}_{2}=7\right.$ e $\mathrm{CC} i_{3}=7$ anos $)$, em relação ao grupo de espécies comerciais $\left(\mathrm{CC} i_{1}=37 ; \mathrm{CC} i_{2}=\right.$ 29 e $\mathrm{CC}_{3}=22$ anos). $\mathrm{O}$ da $M$. huberi foi mais longo que do total da comunidade $\left(\mathrm{CC} i_{1}=30 ; \mathrm{CC} i_{2}=\right.$ 25 e CCi $i_{3}=19$ anos) e menor do que os ciclos do grupo de espécies comerciais (Tabela 2).

Utilizando o ciclo de corte de 35 anos (Tabela 3), a intensidade de corte pelo MMOF, nas diferentes taxas de crescimento, para o total da comunidade em percentual em relação a intensidade praticada $\left(23,00 \mathrm{~m}^{3} \mathrm{ha}^{-1}\right)$ foram de $\mathrm{IC} i_{1}=274,3 \%, \mathrm{IC}_{2}=290,6 \%$ e $\mathrm{IC} i_{3}=308,3 \%$. Para o grupo das espécies comerciais foram de $\mathrm{IC} i_{1}=-3,65 \%, \mathrm{IC}_{2}=13,86 \%$ e $\mathrm{IC} i_{3}=38,6 \%$. Para a $M$. huberi foram de $\mathrm{IC} i_{1}=12,0 \%, \mathrm{IC} i_{2}=29,9 \%$ e $\mathrm{IC} i_{3}=54,1 \%$. A intensidade de corte calculada foi menor que a realizada (valor negativo), somente para a taxa $i_{1}$ no grupo de espécies comerciais.

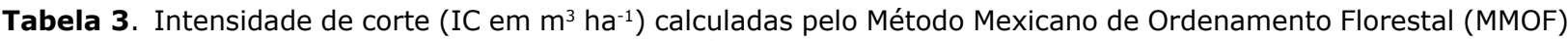
em uma floresta de terra firme na Amazônia Oriental, em um ciclo de corte (CC) de 35 anos. i: taxa de crescimento.

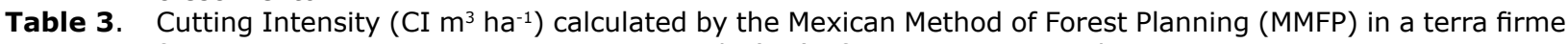
forest in Eastern Amazon, in a cutting cycle (CC) of 35 years. i: growth rate.

\begin{tabular}{lccc}
\hline Espécie & $\mathbf{C C} \mathbf{3 5}$ anos & $\mathbf{I C i}_{3}$ \\
Manilkara huberi (Ducke) A. Chev. & $\mathbf{I C i _ { 1 }}$ & $\mathbf{I C i}_{2}$ & 4,929 \\
Espécies comerciais & 3,582 & 4,154 & 31,888 \\
Total comunidade & 22,162 & 26,188 & 93,908 \\
\hline
\end{tabular}

O volume projetado para os anos de 1998 e 2010 (Figura 2 A) da comunidade total, nas diferentes taxas de crescimento, $\mathrm{Vn} i_{1}=191,689 ; \mathrm{Vn} i_{2}=192,243 ; \mathrm{e} \mathrm{Vn} i_{3}=192,868 \mathrm{~m}^{3} \mathrm{ha}^{-1}$; e Vni $i_{1}=229,409$; $\mathrm{Vn} i_{2}=232,515 ; \mathrm{e} \mathrm{Vni} i_{3}=236,059 \mathrm{~m}^{3} \mathrm{ha}^{-1}$, respectivamente, não diferenciaram-se estatisticamente ( $p>$ $0,05)$ dos volumes observados nas parcelas permanentes. Isso ocorreu também $(p>0,05)$ para o grupo de espécies comerciais (Figura $2 \mathrm{~B})$ em $1998\left(\mathrm{Vn} i_{1}=35,856 ; \mathrm{Vn} i_{2}=36,253 ; \mathrm{e} \mathrm{Vn} i_{3}=36,923 \mathrm{~m}^{3}\right.$ ha $\left.^{-1}\right)$ e em $2010\left(V n i_{1}=42,154 ; V_{2} i_{2}=44,372 ;\right.$ e Vn $\left.i_{3}=48,322 \mathrm{~m}^{3} \mathrm{ha}^{-1}\right)$; e M. huberi (Figura $2 \mathrm{C}$ ) em $1998\left(\mathrm{Vn} i_{1}=5,751 ; \mathrm{Vn} i_{2}=5,814 ; \mathrm{e} \mathrm{Vn} i_{3}=5,913 \mathrm{~m}^{3} \mathrm{ha}^{-1}\right)$ e em $2010\left(\mathrm{Vn} i_{1}=6,859 ; \mathrm{Vn} i_{2}=7,212 ; \mathrm{e} \mathrm{Vn} i_{3}=\right.$ $7,806 \mathrm{~m}^{3}$ ha $\left.^{-1}\right)$.

rciais e para M. huberi estão próximos aos recomendados por diversos estudos para florestas tropicais da Amazônia, para EIR, entre 20 a 40 anos (SILVA, 1992; SILVA et al., 1995; ALDER; SILVA, 2001; AZEVEDO et al., 2008), aplicando a técnica de exploração de impacto reduzido que acelera o crescimento (VIDAL et al., 2002). Utilizando a taxa de crescimento $\left(i_{3}\right)$ o ciclo de corte, como por exemplo, para espécies comerciais que podem ser submetidas a uma nova colheita em 22 anos de forma sustentável, sem impactar nos processos de recuperação.

Considera-se a taxa $i_{3}$ a mais adequada teoricamente por considerar somente a recuperação do volume retirado $(\mathrm{Vr})$, sendo esse o objetivo principal do manejo florestal.

O ciclo de corte mais curto para o total da comunidade nas três taxas de crescimento deve-se ao volume remanescente ser maior, sendo a intensidade de corte baixa ( $\left.23 \mathrm{~m}^{3} \mathrm{ha}^{-1}\right)$ se comparada com o volume inicial, promovendo uma acelerada recuperação com o crescimento maior $\left(3,212 \mathrm{~m}^{-3} \mathrm{ha}^{-1}\right.$ ano $^{-1}$ ) em relação ao grupo das espécies comerciais e da $M$. huberi (Tabela 2). Mas essa recuperação considera as espécies comerciais, não comerciais e potenciais, servindo como indicativo da recuperação acelerada do volume do ecossistema florestal após a EIR. Mazzei et al. (2010) observaram, em uma floresta na Amazônia, com baixa intensidade de exploração, que a recuperação da biomassa que havia antes da exploração, considerando o total da comunidade acima do solo, foi em 15 anos.

Os ciclos de corte calculados pelo MMOF (Tabela 2), considerando o grupo das espécies comeResultados importantes foram obtidos por Reis et al. (2010) que avaliaram através de um acompanhamento de 28 anos após a colheita de alta intensidade $\left(72,5 \mathrm{~m}^{3} \mathrm{ha}^{-1}\right)$, que o volume colhido foi recuperado, principalmente pelas espécies potenciais. Isso é um forte indicativo que as estimativas do ciclo de corte pelo MMOF foram próximas a realidade das florestais tropicais da Amazônia. Entretanto, Reis et al. (2010) observaram que M. huberi não recuperou a densidade e o volume 28 anos após a colheita. Também Gayot e Sist (2004) realizaram simulações para a população de M. huberi, em Paragominas, PA, e registram que na melhor das hipóteses de crescimento $\left(0,63 \mathrm{~cm} \mathrm{ano}^{-1}\right)$ e 
Reis et al. - Aplicação do método mexicano de ordenamento florestal (MMOF)

para estimar ciclo e intensidade de corte em floresta na Amazônia oriental

mortalidade $\left(0,4 \%\right.$ ano $\left.^{-1}\right)$ a taxa de recuperação da densidade inicial em 30 anos seria de apenas 52\%. Apesar disso, na área do presente estudo, Reis et al. (2013) e Reis et al. (2015) observaram que M. huberi apresentou 30\% de aumento da área basal entre 1998 a 2010, indicando uma recuperação após colheita. Pelo MMOF estima-se que recuperação da M. huberi seria em 19 anos na taxa de crescimento $i_{3}$ (Figura 2C).
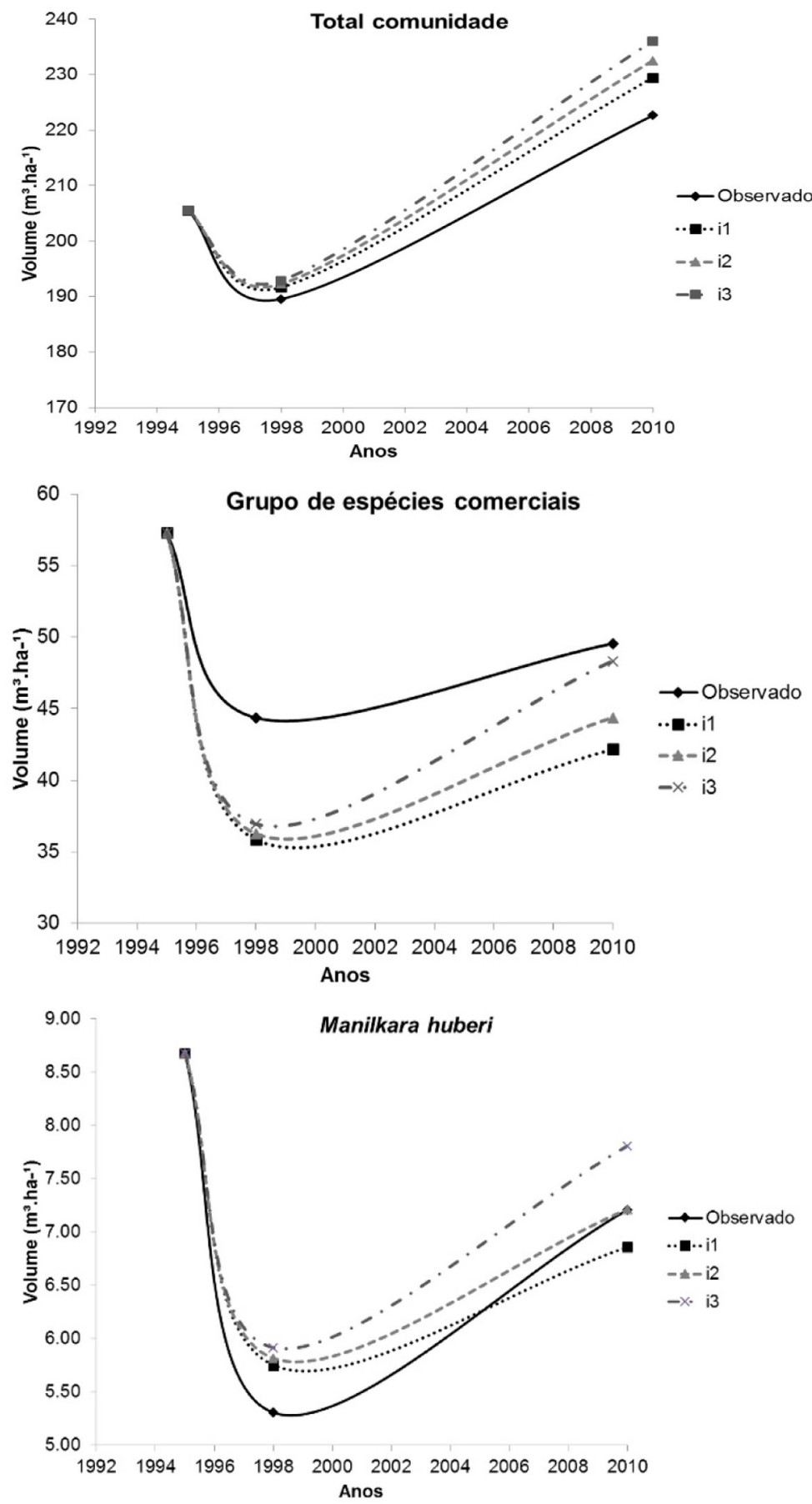

Figura 2. Volumes observados $\left(\mathrm{m}^{3} \mathrm{ha}^{-1}\right)$ e projetados para os anos 1998 e 2010, pelo Método Mexicano de Ordenamento Florestal (MMOF), em uma floresta de terra firme na Amazônia Oriental. A: Total da comunidade; B: Grupo de espécies comerciais; e C: Manilkara huberi (Ducke) A. Chev. i= taxa de crescimento.

Figure 2. Observed volumes $\left(\mathrm{m}^{3} \mathrm{ha}^{-1}\right)$ and projected ones for the years 1998 and 2010 by the Mexican Method of Forest Planning (MMFP) in a terra firme forest in the Eastern Amazon. A: Total of the community; B: Group of commercial; and C: Manilkara huberi (Ducke) A. Chev. $\mathrm{i}=$ growth rate.

Isso indica que a aplicação do MMOF devesse levar em consideração o crescimento do grupo de espécies comerciais, crescimento individual das espécies e características próprias de cada sítio (histórico da área, relevo, solo, clima etc.). 
Considerando o ciclo de corte de 35 anos, a intensidade de corte (IC) calculada pelo MMOF (Tabela 3 ) nas taxas de crescimento $i_{1}, i_{2}$ e $i_{3^{\prime}}$ poderia ter sido maior do que a praticada, menos para a taxa $i_{1}$ em relação as espécies comerciais. A IC $i_{3}$ de $31,888 \mathrm{~m}^{3}$ ha $^{-1}$, máxima possível para o CC de 35 anos nesta floresta, ficou próxima a permitida pela legislação florestal (BRASIL, 2015), máximo $30 \mathrm{~m}^{3} \mathrm{ha}^{-1}$ e o indicado na literatura (ALDER; SILVA, 2001; AZEVEDO et al., 2008). Isso evidencia que o método pode ser usado para os atuais PMFS praticados na Amazônia, como um guia inicial para regulação sustentável da produção de madeiras. Os valores também ficaram próximos aos encontrados por Souza et al. (2004) que recomendaram intensidade de corte de 25 a $35 \mathrm{~m}^{3} \mathrm{ha}^{-1}$, com CCs menores de 17 e 18 anos, avaliando a sustentabilidade econômica (taxas de juros equivalentes à taxa de crescimento da floresta).

As três taxas de crescimento calculadas seguem a tendência da floresta após a colheita. Os volumes projetados pelo MMOF seguiram a tendência até 13 anos da exploração, tanto para a comunidade total (Figura 2A) como para as espécies comerciais (Figura 2B) e M. huberi (Figura 2C) não apresentou diferença estatística a 5\% de probabilidade. Isso demostra que o método para florestais tropicais, após a colheita, pode ser usado para calcular os volumes no final do ciclo de corte.

As intensidades de corte de 30 a $40 \mathrm{~m}^{3} \mathrm{ha}^{-1}$ com ciclo de 30 anos, de acordo com simulação a nível de árvore individual e de povoamento total (ALDER; SILVA, 2001; PHILLIPS et al., 2004; AZEVEDO et al., 2008) tendem a ser sustentáveis em aproximadamente 6 ciclos. Isso porque a produtividade decresce nas colheitas subsequentes e fica abaixo dos $30 \mathrm{~m}^{3} \mathrm{ha}^{-1}$ planejado no primeiro ciclo de corte.

Recomenda-se usar o MMOF para períodos de até um ciclo de corte pela sua característica exponencial, por não ter um momento de desaceleração do crescimento (fase assintótica), por considerar um crescimento constante ao longo do tempo e não considerar as taxas de recrutamento e ingresso, por tanto não é recomendado para projeções de ciclos muito longos ou para vários ciclos de corte. Uma alternativa seria a compatibilização do MMOF com a função logística que apresenta um momento assintótico, o que poderia ser mais realístico biologicamente, para projeção de vários ciclos de corte. Também se recomenda o ajuste de equações de volume específica por espécie e a aplicação de MMOF.

\section{CONCLUSÕES}

O Método Mexicano de Ordenamento Florestal (MMOF) pode ser usado como um método de controle de corte por volume para estimar o ciclo de corte (CC) e intensidade de corte (IC) por um período não superior a 30 ano nos planos de manejo florestal sustentáveis na Amazônia Oriental.

\section{AGRADECIMENTOS}

À Embrapa Amazônia Oriental, pelo apoio dado através do Projeto Manejo Florestal na Amazônia, à CAPES, pela concessão de Bolsa ao primeiro autor, e ao CNPq, pela concessão de Bolsa à terceira autora.

\section{REFERÊNCIAS BIBLIOGRÁFICAS}

ALDER, D.; SILVA, J. N. M. Sustentabilidade da produção volumétrica: um estudo de caso na Floresta Nacional do Tapajós com auxílio do modelo de crescimento Cafogrom. In: SILVA, J. N. M.; CARVALHO, J. O. P.; YARED, J. G. A silvicultura na Amazônia Oriental: Contribuições do Projeto Embrapa/DFID. Belém: Embrapa Amazônia Oriental/DFID. 2001. p. 325-337.

AZEVEDO, P. C. SANQUETTA, C. R.; SILVA, J. N. M.; MACHADO, S. A.; SOUZA, C. R.; OLIBEIRA, M. M. Simulação de estratégias de manejo florestal na Amazônia com o uso do modelo SYMFOR. Acata Amazonica, Manaus, v. 38, n. 1, p. 51-70, 2008. 
Reis et al. - Aplicação do método mexicano de ordenamento florestal (MMOF)

para estimar ciclo e intensidade de corte em floresta na Amazônia oriental

BAIMA, A. M. V.; SILVA, S. M. A.; SILVA, J. N. M. Equações de volume para floresta tropical de terra firme em Moju, PA. In: SILVA, J. N. M.; CARVALHO, J. O. P.; YARED, J. A C. (Eds.). A silvicultura na Amazônia Oriental: contribuições do projeto Embrapa/DFID. Belém: Embrapa Amazônia Oriental - DFID, 2001. p. 367-392.

BRASIL. SEMAS - Secretaria de meio ambiente e sustentabilidade. Instrução Normativa 05. Dispõe sobre procedimentos técnicos para elaboração, apresentação, execução e avaliação técnica de Plano de Manejo Florestal Sustentável - PMFS no estado do Pará. 2015. Disponível em: < https://www.semas.pa.gov. br/2015/09/11/in-05-de-10092015-publicada-no-doe-32969-de-11092015-paginas-de-37-57/ >. Acesso em: 31 ago 2016.

BRAZ, E. M.; SCHNEIDER, P. R.; MATTOS, P. P.; SELLE, G.L.; THAINES, F.; RIBAS, L. A.; VUADEN, E. Taxa de corte sustentável para manejo das florestas tropicais. Ciência Florestal, Santa Maria, v. 22, n. 1, p. 137-145, 2012.

COSTA, D. H. M.; FERREIRA, C. A. P.; SILVA, J. N. M.; LOPES, J. do C. A.; CARVALHO, J. O. P. Potencial madeireiro de floresta densa no município de Moju, estado do Pará. Belém: Embrapa - CPATU, 1998. 33 p. (Documentos Embrapa, 121)

GAYOT, M.; SIST, P. Vulnerabilité des espèces de maçaranduba face à l'exploitation en Amazonie brésilienne: nouvelles normes d'exploitation à definir. Revue Bois et Forêts des Tropiques, Nogent-Sur-Marne, v. 58, n. 208, p. 75-90, 2004.

LOPES, J. C. A.; WHITMORE, T. C.; BROWN, N. D.; JENNING, S. B. Efeito da exploração florestal nas populações de mudas em uma floresta tropical úmida no município de Moju, PA. In: SILVA, J. N. M; CARVALHO, J. O. P.; YARED, J. A. G. (Eds.). A silvicultura na Amazônia Oriental: contribuições do projeto Embrapa/DFID. Belém: Embrapa Amazônia Oriental - DFID, 2001. p. 227-251.

MAZZEI, L.; SIST, P.; RUSCHEL, A.; PUTZ, F.; MARCO, P; PENA, W.; FERREIRA, J. E. R. Above-ground biomass dynamics after reduced-impact logging in the Eastern Amazon. Forest Ecology and Management, Amsterdam, v. 259, n. 3 , p. $367-373,2010$

PHILLIPS, P. D., AZEVEDO C. P., DEGEN, B., THOMPSON, I. S., SILVA, J. N. M., Van GARDINGEN P.R. An individual-based spatially explicit simulation model for strategic forest management planning in the eastern Amazon. Ecological Modelling, Amsterdam, v.173, p. 335-354, 2004.

REIS, L. P.; REIS, PAMELLA C. M.; RUSCHEL, A. R.; SILVA, J. N. M.; CARVALHO, J. O. P.; SOUZA, A. L.; SOARES, M. H. M.; MIYAHARA, R. K. N. Forest dynamics in the eastern Amazon with special reference to Sapotaceae species. Floresta, Curitiba, v. 45, n. 3, p. 567, 2015.

REIS, L. P.; RUSCHEL, A. R.; SILVA, J.N.M.; REIS, P.C.M.; CARVALHO, J.O.P.; SOARES, M.H.M. Dinâmica da distribuição diamétrica de algumas espécies de Sapotaceae após exploração florestal na Amazônia Oriental. Revista de Ciências Agrárias, Belém, v. 57, n. 3, p. 234-243, 2014 a.

REIS, P. C. M.; REIS, L. P.; RUSCHEL, A. R.; SILVA, J.N.M.; CARVALHO, J.O.P.; QUEIROZ, W. T. Effect of timber harvesting on density and basal area of Lecythidaceae species in the eastern Amazon. Floresta, Curitiba, v. 44, n. 2, p. 229, 2014b.

REIS, L. P.; SILVA, J. N. M.; REIS, P. C. M.; CARVALHO, J. O. P.; QUEIROZ, W. T.; RUSCHEL, A. R. Efeito da exploração de impacto reduzido em algumas espécies de Sapotaceae no leste da Amazônia. Floresta, Curitiba, v. 43, n. 3, p. 395, 2013.

REIS, L. P.; RUSCHEL, A. R; COELHO, A. A.; LUZ, A. S.; MARTINS-DA-SILVA, R. C. V. Avaliação do potencial madeireiro na Floresta Nacional do Tapajós após 28 anos da exploração florestal. Pesquisa Florestal Brasileira, Cascavel, v. 30, n. 64, p. 265-281, 2010. 
ROJO, J. M. T. Sostenibilidad del volumen de cosecha calculado con el Método Mexicano de Ordenación de Montes. Madera y Bosques, Xalapa, v. 6, n. 2, p. 57-72, 2000.

SILVA, J. N. M. A note on Brazil's tropical rain forests under new Forest management regulations. Journal of tropical forest Science, Malaysia, v. 4, n. 4, p. 355-356, 1992.

SILVA, S. M. A. S.; SILVA, J. N. M.; BAIMA, A. M. V.; LOBATO, N. M.; THOMPSON, I. S.; COSTA FILHO, P. P. Impacto da exploração madeireira em floresta de terra firme no município de Moju, estado do Pará. In: SILVA, J. N. M; CARVALHO, J. O. P.; YARED, J. A. G. (Eds.). A silvicultura na Amazônia Oriental: contribuições do projeto Embrapa/DFID. Belém: Embrapa Amazônia Oriental - DFID, 2001. p. 227-251.

SILVA, J. N. M; DE CARVALHO, J. O. P.; LOPES, J. C. A.; ALMEIDA, B. F.; COSTA, D. H. M.; OLIVEIRA, L.C.; VANCLAY, J. K.; SKOVSGAARD, J. P. Growth and yield of a tropical rain forest in the brazilian Amazon 13 years after logging. Forest Ecology and management, Amsterdam, v.71, n.3, p.267-274, 1995.

SOUZA, A. L.; SOARES, C. P. B. Florestas nativas: estrutura, dinâmica e manejo. Viçosa: Editora UFV, 2013, $322 \mathrm{p}$.

SOUZA, D. R.; SOUZA, A. L.; SILVA, M. L.; RODRIGUES, F. L. Ciclo de corte econômico ótimo em floresta ombrófila densa de terra firme sob manejo florestal sustentável, Amazônia Oriental. Revista Árvore, Viçosa, v.28, n.5, p.681-689, 2004.

VIDAL, E.; VIANA, V; BATISTA, J. L. F. Crescimento de floresta tropical três anos após colheita de madeira com e sem manejo florestal na Amazônia oriental. Scientia Forestalis, Piracicaba, v.16, n. 61, p. 133-143, 2002.

Recebido em 07/03/2016

Aceito para publicação em 21/09/2016

Sci. For., Piracicaba, v. 45, n. 113, p. 187-195, mar. 2017

DOI: dx.doi.org/10.18671/scifor.v45n113.19 